\title{
Scleral Fixation of a Toric Lens to Treat Corneal Astigmatism in Eyes without Capsular Support
}

\author{
Matthew S Ward' \\ Andrew $\mathrm{C} \mathrm{Hou}{ }^{2}$ \\ David A Murphy ${ }^{2}$ \\ Mason A Schmutz ${ }^{3}$ \\ Kamran M Riaz $\mathbb{D}^{2}$ \\ 'Riverwoods Eye Center, Provo, UT, \\ USA; ${ }^{2}$ Dean McGee Eye Institute, \\ University of Oklahoma, Oklahoma City, \\ OK, USA; ${ }^{3}$ Excel Eye Center, Saratoga \\ Springs, UT, USA
}

Purpose: To describe surgical technique and report short-term visual outcomes after suturefixation of a single-piece eyelet-toric (SET) intraocular lens (IOL) for treatment of concurrent aphakia and astigmatism.

Design: Retrospective, noncomparative, and non-consecutive case series.

Methods: This was a case series of eleven eyes who underwent successful SET. Eligible eyes had loss of capsular support or aphakia with a minimum of symmetric corneal astigmatism 1.75 diopters (D). Outcome measures included uncorrected distance visual acuity (UDVA), corrected distance visual acuity (CDVA), magnitude of residual refractive cylinder, and complications resulting from the SET procedure.

Results: Preoperative UDVA and CDVA in logMAR scale were 1.46 and 0.45 , respectively. Mean preoperative keratometric and refractive cylinder were $3.67 \mathrm{D}$ and $2.52 \mathrm{D}$, respectively. Postoperative UDVA and CDVA were 0.51 and 0.27 , respectively, three months after surgery (POM3). Residual refractive cylinder at POM3 was $0.93 \mathrm{D}$.

Conclusion: SET technique reduced refractive cylinder and improved UDVA and CDVA. SET may be adapted by surgeons using a readily available IOL and familiar scleral-fixation maneuvers.

Keywords: aphakia, toric, suture fixation, secondary lens, astigmatism

\section{Plain Language Summary}

When patients undergo cataract surgery, an intraocular lens (IOL) implant is placed in the eye in order to improve patients' visual acuity. In certain cases, such as after trauma or complex cataract surgery, an IOL cannot be implanted in the usual technique and instead must be suture-fixated into the eye. While these suture fixated IOLs (SFIOLs) can improve patients' vision after surgery, no techniques exist to concurrently correct astigmatism at the time of SFIOL surgery. We describe a unique SFIOL technique that can also correct patients' astigmatism that has not been previously published in the literature. This technique involves modifications to techniques already used by surgeons who perform SFIOL surgery, thus making it easily adaptable Additionally, we report the refractive outcomes of 11 patients who underwent this novel surgical procedure.

\section{Introduction}

Recent years have seen the introduction of various techniques for the surgical management of aphakia without capsular support. Traditional surgical approaches in these cases utilize sutures (polypropylene or polytetrafluoroethylene (Gore-Tex, W.L. Gore, Newark, Delaware)) for scleral fixation of intraocular lenses (IOLs). ${ }^{1,2}$ Other scleral fixation IOL (SFIOL) surgical techniques, such as intra-scleral haptic 
fixation (ISHF), utilize glued flaps or cauterized haptics to fixate the IOL to the sclera. ${ }^{3}$ Each of these techniques has advantages and drawbacks and anterior segment surgeons have adopted these procedures based on personal preference and comorbid ocular conditions. In the United States, no current technique in wide use provides surgeons with the ability to correct astigmatism at the IOL plane at the time of SFIOL surgery, despite the substantial corneal cylinder frequently encountered in aphakic patients.

Broad use of toric IOL technology in eyes with good capsular support has been well established since FDA approval in 2005. Successful transscleral fixation to stabilize previously implanted intracapsular toric IOLs has also been described. ${ }^{4}$ However, more than fifteen years later there has been minimal utilization of toric IOL technology to treat astigmatism in eyes without capsular support.

The concept of transscleral Gore-Tex fixation of the MX60 IOL (Bausch \& Lomb, Rochester, New York) has previously been described in the literature as an effective approach for aphakia. ${ }^{5,6}$ With this technique, the haptics are trimmed and Gore-Tex suture is threaded through eyelets at the optic-haptic junction and externalized to achieve secure four-point transscleral fixation with good refractive outcomes. $^{7}$

We postulated that this existing SFIOL technique could be modified using a sutured eyelet-toric (SET) IOL to treat aphakia while concurrently reducing postoperative refractive cylinder. We describe the SET technique and report a case-series of 11 patients who underwent uneventful SET with at least 3-months of follow up.

\section{Materials and Methods}

The study received retrospective multi-center case review approval by the Institutional Review Board (IRB) at the University of Oklahoma Health Science Center (IRB: 12428) to investigate the safety and efficacy of SET in the treatment of corneal astigmatism. All clinical procedures were conducted according to the principles of the Declaration of Helsinki. Informed consent was obtained from all patients for the SET procedure. The following data were collected: age, gender, indication for initial surgery, previous ocular history, pre- and postoperative corrected distance visual acuity (CDVA), preoperative corneal cylinder, postoperative refractive cylinder, complications, the operated eye, and surgical technique.

Inclusion criteria for the study were successful completion of the SET procedure with at least 3 months of postoperative follow up. Candidates for the SET procedure had
(1) symmetrical corneal astigmatism greater than $1.75 \mathrm{D}$, (2) current aphakia, dislocated IOL, or subluxed crystalline lens, and (3) significant visual potential defined as improvement in distance visual acuity with preoperative refraction. Patients with irregular corneal astigmatism requiring correction with hard contact lenses, or patients requiring penetrating keratoplasty or lamellar keratoplasty were not eligible for the procedure. All patients underwent surgery between 2019 and 2020. All patients underwent a standard ophthalmologic examination including measurements of uncorrected distance visual acuity (UDVA) and CDVA with a Snellen chart at 20 feet, slit-lamp examination, measurement of IOP, dilated indirect slit-lamp biomicroscopy and topography.

Preoperative biometry was performed in all cases using either the IOLMaster 700 (Version 1.80.10.61129; Carl Zeiss Meditec, Dublin, CA) or Lenstar (EyeSuite i8.0.0.0, Haag-Streit, Köniz, Switzerland). The preoperative biometry calculations were reviewed and the axial length, keratometry, and corneal astigmatism were recorded. Astigmatism was additionally assessed using a corneal topography unit to corroborate the steep meridian and magnitude of keratometric astigmatism as measured by optical biometry. The Galilei device (Ziemer, Biel, Switzerland) was by MJW and MAS, and the Atlas 9000 device (Carl Zeiss Meditec, Dublin, CA) was used by KMR. Unless a significantly near target was requested by the patient (Case 8), a target spherical equivalent refraction of -0.50 to -1.50 was chosen with the expectation of an "in-the-bag" effective lens position. The online Barrett Toric Calculator 2.0 was used in all cases to calculate the power of the intended IOL and meridian location of IOL fixation. Surgically induced astigmatism (SIA) value of 0.4 was used in all cases. Postoperative measurements were taken after suture removal in all cases.

\section{Surgical Procedure}

The previously described sutured MX60 surgical technique was modified in consideration of a toric IOL and can be found online (https://www.youtube.com/watch?v= gUXJZ5FSMO4). Of note, the use of Gore-Tex suture for ophthalmic surgery is considered off-label. Preoperatively, toric marks are placed at the limbus to demarcate the vertical and horizontal meridians. Intraoperatively, a toric marker is used to stain the cornea at two points $180^{\circ}$ apart on the steep keratometric meridians. An anterior chamber maintainer or pars plana infusion cannula is placed. If vitreous is present, anterior or 
pars-plana vitrectomy is performed. Limited conjunctival peritomies are made adjacent to the corneal marks. Two tandem anterior sclerotomy sites are marked with calipers $1.5 \mathrm{~mm}$ posterior to the limbus radially in line with steep meridian. An additional two tandem posterior sclerotomy sites are radially placed $1.5-2.0 \mathrm{~mm}$ posterior to the anterior sclerotomy sites. A 23-gauge needle is then used to create four sclerotomy wounds. Prior to insertion, the MX60T IOL haptics are trimmed distal to the eyelet to minimize potential for iris chafing, allow for radial suture placement, and decrease risk of entanglement during IOL insertion and suture externalization. Two generous lengths of 7-0 Gore-Tex suture (CV-8) are cut from the needles. Each length of suture is threaded through its corresponding eyelet of the MX60T IOL creating four free ends of suture (two on each side).

At this juncture, the surgeon can insert the IOL and externalize the sutures in one of two methods. In the first method (used by MSW and MAS), the pre-threaded IOL is then folded into a standard cartridge and advanced with care to ensure the sutures glide freely. The IOL-suture complex is inserted through a $2.4 \mathrm{~mm}$ incision and all four ends of the suture are externalized through their corresponding sclerotomies using 25 $\mathrm{g}$ microforceps. In the second method (used by $\mathrm{ACH}$ and KMR), all 4 sutures are externalized prior to IOL insertion; the wound is then enlarged to approximately $4.0 \mathrm{~mm}$ and the IOL is placed without the use of a cartridge. Interrupted sutures using 10-0 nylon are used to close the main wound. The online video demonstrates both styles of insertion and externalization methods.

In both methods, the IOL is then centered, and after ensuring the infusion is at or slightly above physiologic IOP, the sutures are tied using a 1-1-1 technique with care to avoid over-tightening the sutures. A slipknot is made on the second throw to enable titration of proper suture tension on both sides. The surgeon must give additional attention to ensure there is equal radial force tension on both sides. When proper tension is obtained, the final locking throw is made. The knots are trimmed and rotated into the eye and the conjunctival peritomy is then closed using fibrin sealant glue (Tisseel; Baxter, Deerfield, IL). The infusion cannula is removed, and all wounds are tested for their integrity and sutured if needed. Figure 1 shows the postoperative appearance of the SET IOL in situ in front and downgaze.

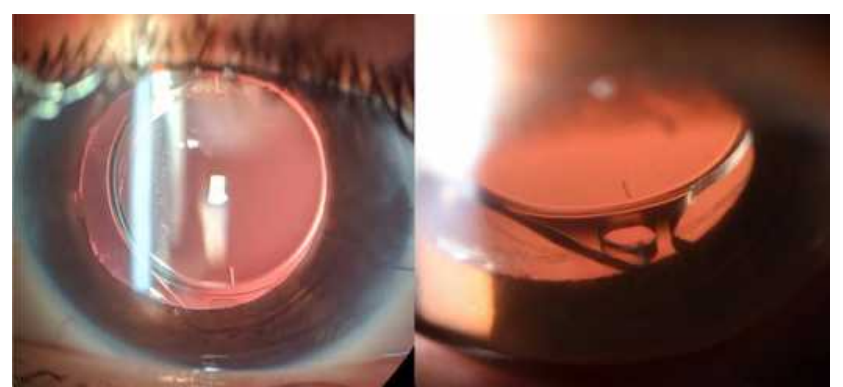

Figure I Postoperative slit-lamp photo of a sutured MX60T IOL in situ. In downgaze, the Gore-tex is visible looping through the eyelet. Note the lack of eyelet distortion indicating proper suture tension.

\section{Results}

Eleven eyes of eleven separate patients were included in the study. Patient demographics are summarized in Table 1. Indications for surgery included dislocated IOL $(n=5$, $45 \%$ ), aphakia ( $n=5,45 \%$ ), and malpositioned anterior chamber IOL $(n=1,9 \%)$. As shown in Table 2, Preoperative mean $\operatorname{logMAR}$ UDVA and CDVA was 1.46 and 0.45 , respectively. Postoperative month 3 mean UDVA and CDVA was 0.51 and 0.27 , respectively. The mean preoperative keratometric astigmatism was $3.66 \mathrm{D}$, refractive astigmatism was $2.52 \mathrm{D}$, and predicted residual cylinder after SET was 0.77 D. UDVA, CDVA and postoperative keratometric astigmatism values were recorded after all corneal sutures were removed. Postoperatively, no rotation of the toric IOL was noted in all cases. Clinically, while no significant IOL tilt was observed at the slit-lamp, diagnostic imaging to assess IOL tilt was not performed in this series. Figure 2 displays preoperative corneal astigmatism and postoperative refractive astigmatism at the spectacle plane. All 11 patients had significantly less refractive astigmatism at the spectacle plane compared to preoperative keratometric astigmatism after the SET procedure. Figure 3 shows double-angle astigmatism plots comparing the preoperative keratometric astigmatism and postoperative refractive astigmatism. Figure 4 displays the postoperative refractive astigmatism prediction error. Of note, measured average residual cylinder after SET in the third postoperative month measured $0.93 \mathrm{D}$, which was significantly lower than preoperative cylinder $(\mathrm{p}<0.05)$ and very close to the predicted residual cylinder value of $0.77 \mathrm{D}$.

In this series, 2 of out 11 patients had postoperative hypotony at the first-day postoperative visit that resolved within the first postoperative week. Vitreous hemorrhage occurred at postoperative month one in one patient (Case 1) with a history of severe penetrating trauma. Vitrectomy was required to evacuate the hemorrhage and it was not felt that 
Table I Patient Demographic Information, Past Ocular History and Indication for SET Surgery

\begin{tabular}{|c|c|c|c|}
\hline Case & $\begin{array}{l}\text { Genderl } \\
\text { Age }\end{array}$ & Past Ocular History & $\begin{array}{l}\text { Diagnosis Prior to } \\
\text { SET Surgery }\end{array}$ \\
\hline I* & $M / 35$ & Open globe injury with subsequent PPV and PPL & Aphakia \\
\hline $2 * *$ & $M / 30$ & Traumatic cataract with subsequent PPV, placement of sulcus IOL & $\begin{array}{l}\text { Dislocation of sulcus } \\
\qquad \text { IOL }\end{array}$ \\
\hline 3 & $\mathrm{~F} / 56$ & $\begin{array}{l}\text { Complex cataract surgery with subsequent PPV, placement of sulcus IOL. History of DME with } \\
\text { focal laser treatment and anti-VEGF injections }\end{array}$ & $\begin{array}{l}\text { Dislocation of sulcus } \\
\qquad \text { IOL }\end{array}$ \\
\hline 4 & $\mathrm{M} / 70$ & Complex cataract surgery with placement of $\mathrm{AClOL}$ & Malpositioned ACIOL \\
\hline 5 & $M / 64$ & Open globe injury with subsequent PPV, PPL, and GDD placement & Aphakia \\
\hline 6 & $M / 75$ & Traumatic injury in context of previous pseudophakia (I-piece IOL) with vitreous prolapse & $\begin{array}{l}\text { Dislocation of I-piece } \\
\qquad \text { IOL }\end{array}$ \\
\hline 7 & $M / 79$ & Complex cataract surgery with aphakia and subsequent PPV & Aphakia \\
\hline 8 & $M / 53$ & Progressive dislocation of I-piece toric IOL & $\begin{array}{l}\text { Dislocation of I-piece } \\
\quad \text { toric IOL }\end{array}$ \\
\hline 9 & $\mathrm{~F} / 67$ & $\begin{array}{l}\text { Previous DSAEK; subsequent trauma with posterior dislocation of I-piece IOL requiring PPV and } \\
\qquad \mathrm{IOL} \text { removal }\end{array}$ & Aphakia \\
\hline 10 & $M / 70$ & Previous PPV; subsequent dislocation I-piece IOL; history of AMD with geographic atrophy & $\begin{array}{l}\text { Dislocation of I-piece } \\
\qquad \text { IOL }\end{array}$ \\
\hline $11 * *$ & $M / 17$ & Traumatic cataract with subsequent PPV and PPL & Aphakia \\
\hline
\end{tabular}

Notes: *This patient required pars plana vitrectomy to evacuate postoperative hemorrhage. **These patients had postoperative hypotony that resolved by one week after surgery.

Abbreviations: PPV, pars plana vitrectomy; PPL, pars plana lensectomy; DME, diabetic macular edema; IOL, intraocular lens; ACIOL, anterior chamber intraocular lens; GDD, glaucoma drainage device (Ahmed tube shunt); DSAEK, descemet's stripping automated endothelial keratoplasty.

the SET IOL surgery or IOL position was responsible for the hemorrhage. The SET procedure was performed in this patient, despite the previous ocular history, because the patient demonstrated significant improvement in visual acuity with preoperative refraction. Following the vitrectomy, the IOL in that patient remained stable for a total of 18 months without recurrent bleeding or inflammation. Unfortunately, this same patient suffered a recurrent retinal detachment at postoperative month six. The detachment was not deemed to be direct sequelae of the SET IOL procedure, but rather longstanding PVR from his original injury. There was no evidence of uveitis-glaucoma-hyphema syndrome, IOL eyelet fracture, or dislocation of the IOL in any patient at the time of last follow-up.

\section{Discussion}

Transscleral suture fixation of the MX60 IOL through its eyelets has been previously described to be a safe and effective technique in eyes without adequate capsular support. $^{6,7}$ Among its advantages, this technique uses a readily available, aberration-neutral, foldable hydrophobic acrylic IOL that can be implanted without scleral flaps. Its hydrophobicity renders it much less likely to opacify than its hydrophilic predecessor Akreos (AO60, Bausch and Lomb, Inc.), which is an important consideration, especially for corneal transplant surgeons, as these complex eyes may require combined or future retinal or endothelial keratoplasty procedures where intraocular gases may be used. ${ }^{8,9}$

In this study, we report a simple modification of this technique wherein suture fixation of an eyelet-toric IOL (SET) can be effectively used to reduce postoperative refractive cylinder in aphakic patients with corneal astigmatism. Pan et al recently reported a technique using polypropylene suture to scleral fixate the haptics of a single-piece acrylic IOL without eyelets (AcrySof Toric SN6AT, Alcon, Fort Worth). ${ }^{10}$ Because the location of fixation did not align with the steep meridian in this technique, a surgically challenging 53-degree counterclockwise rotation from the toric markings was required 


\begin{tabular}{|c|c|c|}
\hline & 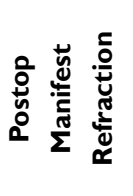 & 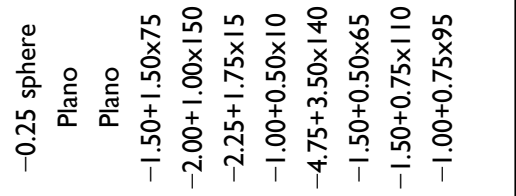 \\
\hline & 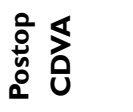 & 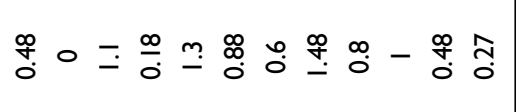 \\
\hline & 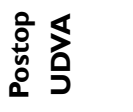 & 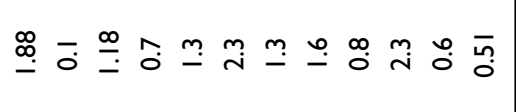 \\
\hline & 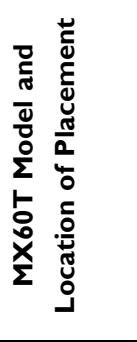 & 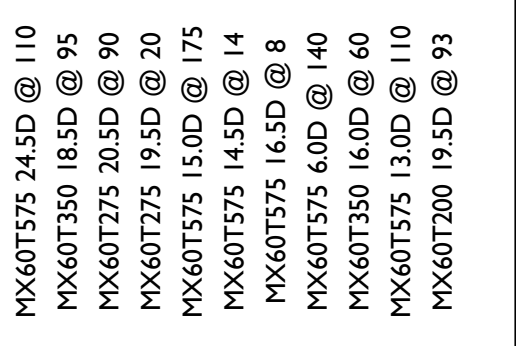 \\
\hline & 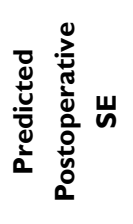 & 盀节 \\
\hline & 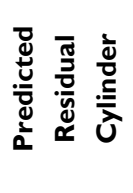 & 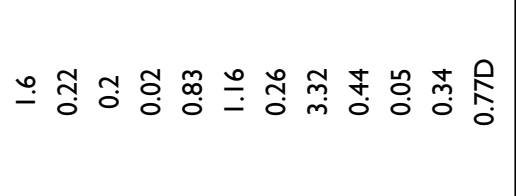 \\
\hline & 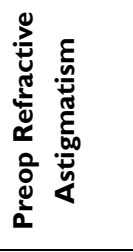 & 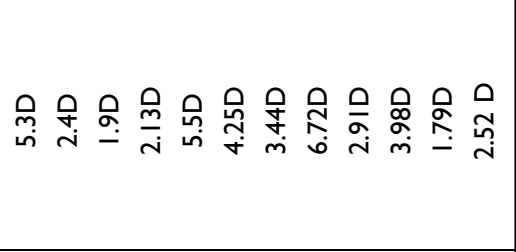 \\
\hline & 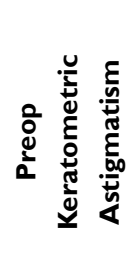 & 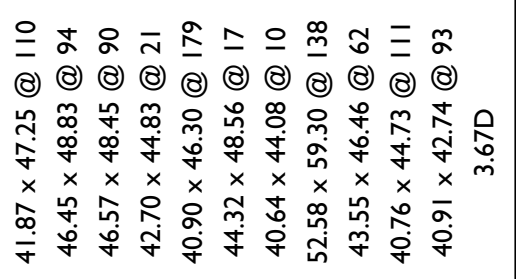 \\
\hline & : & 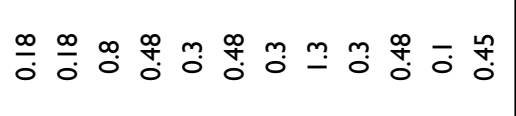 \\
\hline & 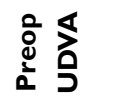 & 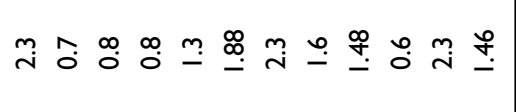 \\
\hline & ฝે & 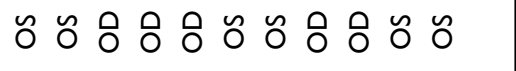 \\
\hline & ்ֻ & 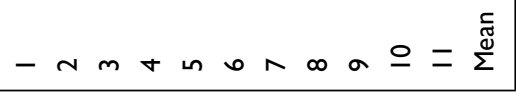 \\
\hline
\end{tabular}

to achieve proper alignment in the steep corneal meridian. Conversely, the eyelet-toric IOL has marks that are in linear alignment with the eyelets to facilitate radial fixation (Figure 5). This alignment permits simple and accurate surgical placement at the steep corneal meridian without the need for challenging rotational corrections. Moreover, unlike placement of a toric IOL in the capsular bag, proper suture fixation eliminates the possibility of postoperative rotation. It should also be mentioned our described SET technique utilizes radial suture placement, allowing for passive, "fool-proof" orientation of the IOL parallel to the iris. Other surgeons have reported scleral fixation of the single-piece eyelet IOL using sclerotomies place tangential to corneal center. ${ }^{11}$ When sutures are tangentially placed, the IOL naturally assumes an orientation perpendicular to the iris. In order to prevent this catastrophic malrotation, the haptics are left in place and carefully positioned behind the sutures to prevent IOL malrotation into a position perpendicular to the iris. To avoid this issue altogether, we strongly recommend radial sclerotomy placement when utilizing the SET technique.

Short-term results in our study population demonstrated that SET may be a viable procedure for the treatment of concurrent aphakia and corneal astigmatism that utilizes surgical maneuvers and materials familiar to surgeons who perform SFIOL surgery. While other SFIOL techniques can achieve reliable refractive outcomes in aphakic patients, these techniques do not address corneal astigmatism. Internationally, surgeons may utilize IOLs such as iris-claw toric IOLs and specially designed SFIOLs (eg, Carlevale IOL, Soleko, Italy); however, these are not available for surgeons to use in the United States. Other astigmatism correction strategies may be employed as an adjunct to these techniques, such as a bioptics approach with staged excimer laser refractive surgery after IOL surgery. However, these approaches may incur additional risk and significant financial costs.

It is important to note that the primary goal of SET is not to obtain spectacle independence as with routine intracapsular toric IOL placement, even though some patients did achieve this result. We emphasize that our surgical results should not be compared to other in-the-bag toric IOL studies published in the literature. Rather, the primary goal of SET is to improve postoperative refractive astigmatism and visual acuity in patients with aphakia and significant corneal astigmatism. In our study population, $82 \%$ of eyes had $>2.0$ D of preoperative corneal 


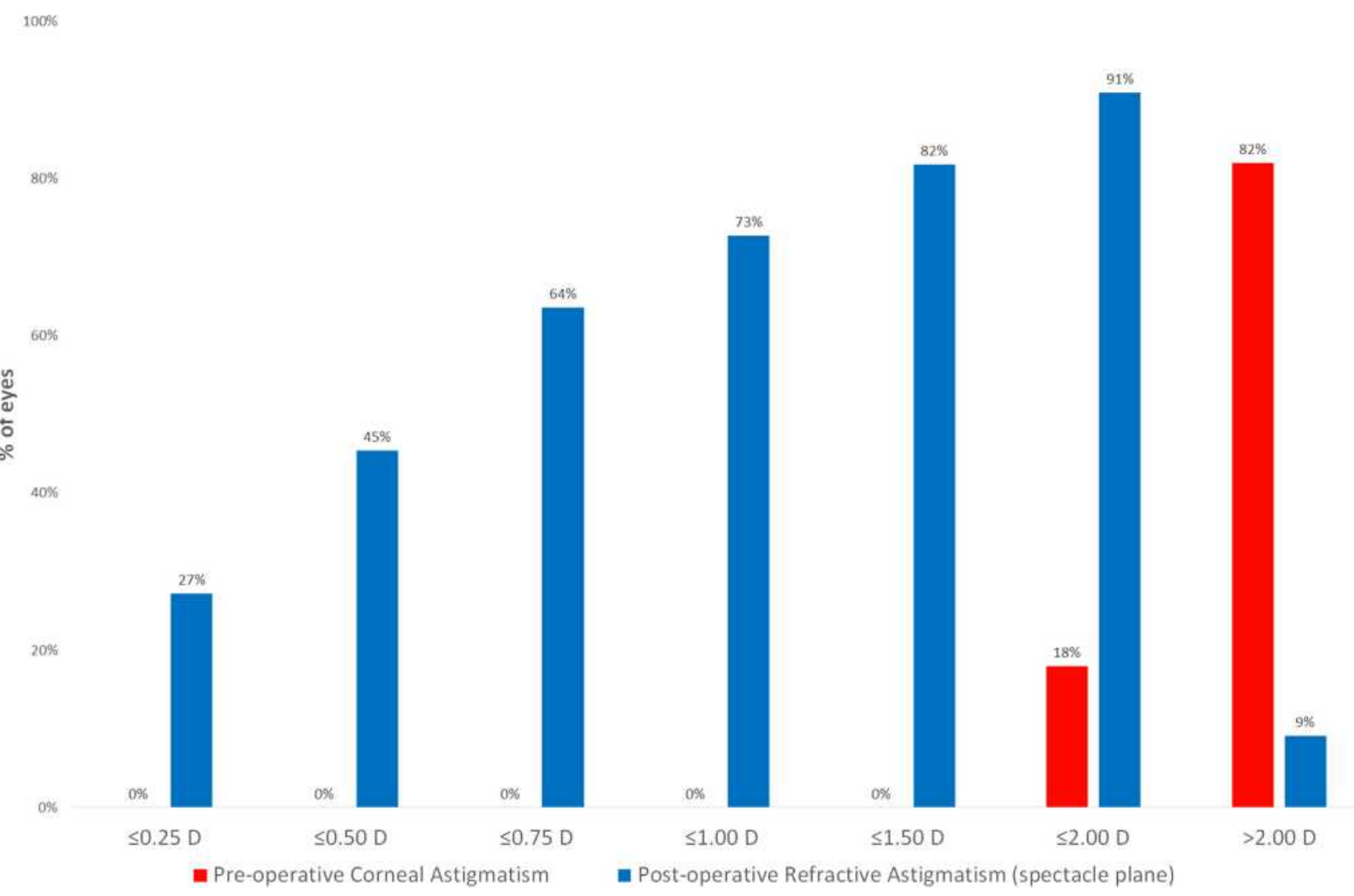

Figure 2 Bar graph comparing preoperative corneal astigmatism to postoperative refractive astigmatism at the spectacle plane.

astigmatism and $50 \%$ had $4.0 \mathrm{D}$ or more requiring the use of the maximum available cylinder power of $5.75 \mathrm{D}$. The benefits of correcting high cylindrical error in these patients are evident by that fact that SET not only improved UDVA, but CDVA as well.

This study has several limitations. First, the SET surgical technique varied slightly between surgeons and thus we were unable to determine the exact amount of surgeon-induced astigmatism (SIA) for this technique, including optimal SIA values for toric IOL calculators. An SIA value of 0.4 was used in all cases. The true magnitude and location of SIA with SET is an important point for future studies to better assess the refractive accuracy of this procedure. Second, though not observed in the present series, scleral fixation with sutures has several potential complications, including suture breakage. ${ }^{8}$ Third, haptic trimming could in theory cause iris chafing and inflammation from the cut edge. We advise haptic trimming as a modification to reduce the risk of suture tangling and iris chafing from the haptics. However, this creates a cut edge which itself may potentially cause mechanical damage to the iris and ciliary body complex. In our series, we noted no cases of prolonged intraocular inflammation or uveitisglaucoma-hyphema syndrome and no transillumination defects related to the IOL. Longer follow up studies are needed to determine long-term viability of the modified haptic edge in these eyes. Fourth, the use of fibrin glue incurs additional costs; however, we postulate that fibrin glue facilitates conjunctival closure, obviates the need to suture conjunctiva, and may also help reduce postoperative hypotony by aiding in sclerotomy closure. It is not necessary to use fibrin glue for the SET procedure as conjunctival closure may be achieved with additional sutures. Fifth, while a 23-gauge needle was used to facilitate externalization of the sutures, this may have led to the postoperative hypotony seen for one week in two cases. It is possible that this hypotony, despite resolving by one week, may affect the final visual acuity. Smaller sclerotomy wounds or use of Hoffman style scleral pockets with $10-0$ nylon suture snares may reduce this transient complication. Sixth, while IOL tilt was assessed clinically, formal image-based assessment, such as with Scheimpflug imaging, anterior segment optical coherence tomography (AS-OCT) or ultrasound biomicroscopy (UBM), was not performed in this series. 


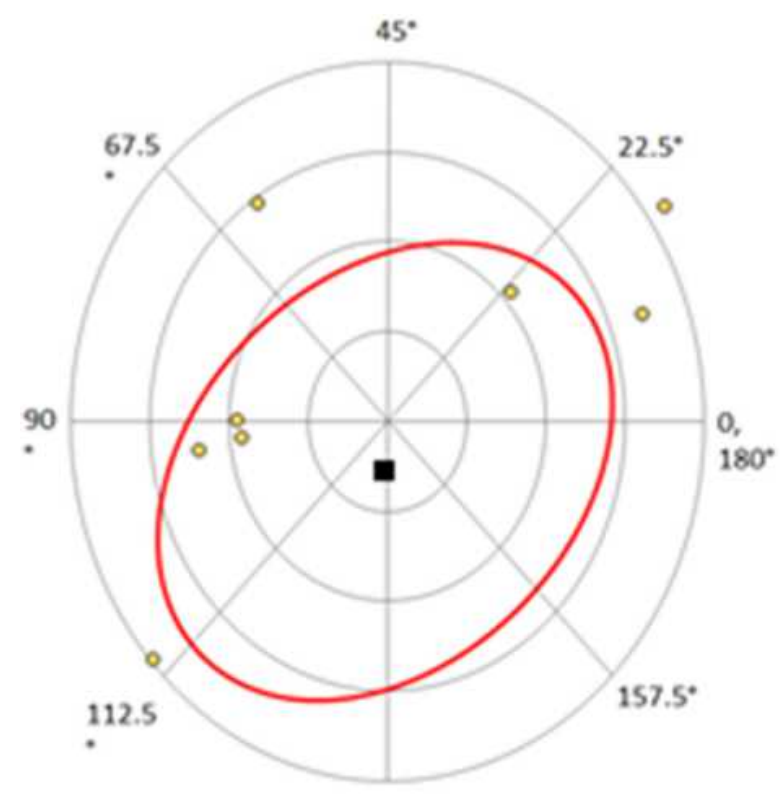

Centroid $0.560 @ 133^{\circ} \pm 4.130$

Mean $\quad 3.660 \pm 1.560$

Centroid

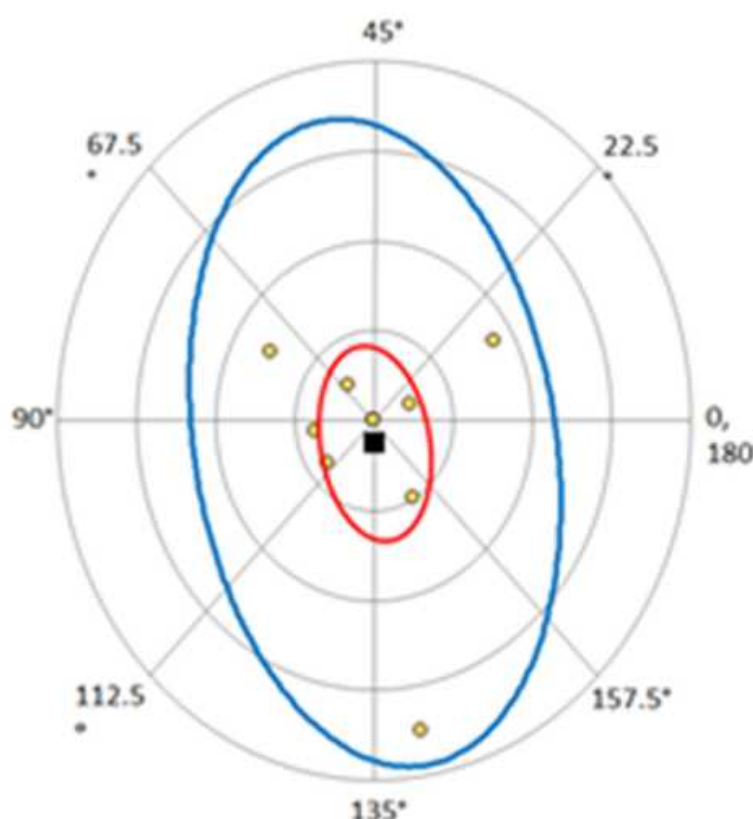

Centroid : $0.250 @ 137^{*} \pm 1.400$

Mean

$0.930 \pm 0.98 D$

Eachrieg $=2.000$

Figure 3 Pre- and post-operative double astigmatism plots.

\section{Postoperative Refractive Astigmatism prediction error (spectacle plane)}

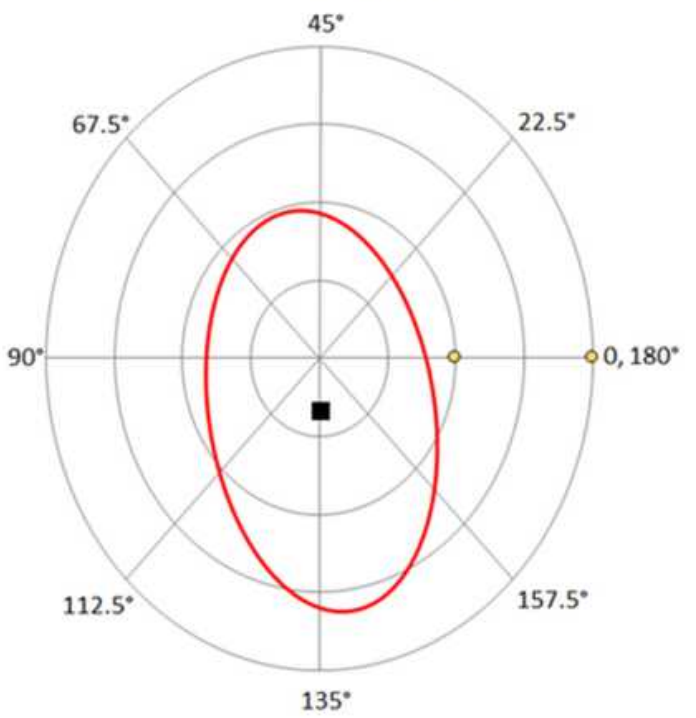

Centroid :0.34D@1374 1.66D

Mean Absolute: $1.28 \pm 0.94 D$

Centroid

$95 \%$ corfidence ellipse of the centroid

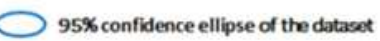

Figure 4 Postoperative refractive astigmatism prediction error (spectacle plane).
Finally, and perhaps most significant to the discussed IOL platform, is the potential for eyelet fracture with overtightening as reported by Watane et al. ${ }^{12}$ In this study, the authors note that the original MX60 and MX60T have been replaced with MX60E and MX60ET, which have lower tensile strength at the eyelet and may result in a higher rate of fracture. Pollman et al compared the eyelet strength of MX60 and MX60E and found that that the MX60 was 67\% stronger, though the result was not statistically significant. ${ }^{13}$ Of interest, both MX60 and MX60E were stronger than or equal to the eyelet of the CZ70BD IOL (Alcon, Ft. Worth), another popularly used IOL for SFIOL surgery. The authors feel that the SET procedure can be safely performed with the softer material, though care and caution are imperative. We emphasize that careful titration of radial suture tension using slipknots while the eye is at a physiologic intraocular pressure or higher may help reduce the risk of this problem. If the eye is soft when the sutures are locked, the chord length of the Gore-Tex/IOL span will increase as pressure rises placing strain on the eyelets. Future studies with larger numbers and longer follow-up may be helpful in determining the longterm viability of the SET procedure as well as the true incidence of eyelet fracture. Ideally, development of an IOL 


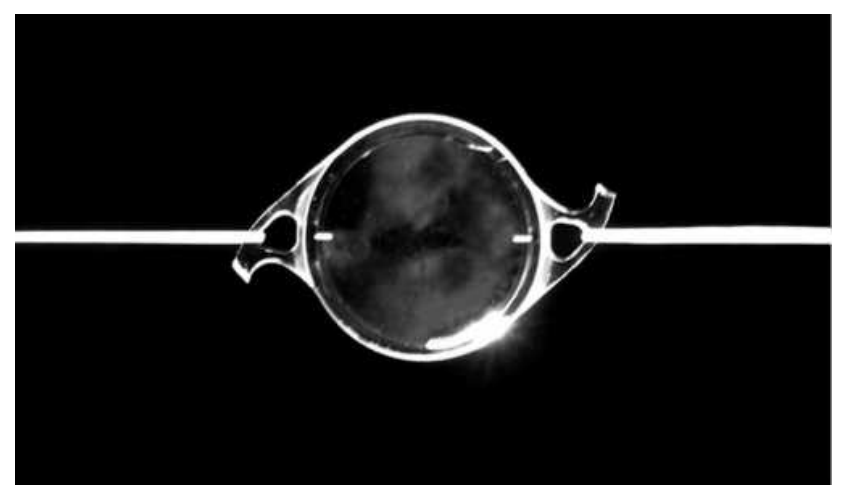

Figure 5 En face view of a threaded Gore-Tex/MX60T complex demonstrating linear alignment of toric marks with the sutures.

designed specifically for transscleral fixation utilizing a large-diameter, foldable hydrophobic acrylic optic with rounded edges, toric capability and ultra-strong, smoothended eyelets without haptics may further improve upon the success of the SET technique.

\section{Conclusion}

In summary, we postulate that anterior segment surgeons using a readily available IOL and familiar scleral fixation maneuvers can easily adopt the described SET technique to correct both aphakia and corneal astigmatism in eyes without capsular support. In our series, patients who underwent SET had good short-term uncorrected and corrected refractive outcomes without significant procedurerelated complications. We offer SET as a new procedure that fulfills a need in a patient population for which no surgical procedure was previously available.

\section{Value Statement}

\section{What Was Known}

Transscleral fixation of secondary posterior chamber IOLs is a safe, reliable and effective way of treating spherical refractive error in patients with aphakia or dislocated IOLs. Current broadly employed fixation techniques do not permit correction of corneal astigmatism at the IOL plane.

\section{What This Paper Adds}

SET is a simple and effective modification of a current IOL fixation technique that achieved significant IOL-based correction of corneal astigmatism resulting in improved UDVA and CDVA in a small series of patients.

\section{Disclosure}

The authors have not received grant support, research funding, nor have any proprietary interests in the materials described in the article.

\section{References}

1. Gess LA. Scleral fixation for intraocular lenses. $J$ Am Intraocul Implant Soc. 1983;9(4):453-456. doi:10.1016/S0146-2776(83)80090-1

2. Khan MA, Gupta OP, Smith RG, et al. Scleral fixation of intraocular lenses using Gore-Tex suture: clinical outcomes and safety profile. $\mathrm{Br}$ J Ophthalmol. 2016;100(5):638-643. doi:10.1136/bjophthalmol-2015306839

3. Yamane S, Ito A. Flanged fixation: Yamane technique and its application. Curr Opin Ophthalmol. 2021;32(1):19-24. doi:10.1097/ ICU.0000000000000720

4. Arjmand P, Chan TYB, Ahmed IIK. Transscleral suture fixation following recurrent toric intraocular lens rotation. $J$ Cataract Refract Surg. 2015;41(5):912-917. doi:10.1016/j.jcrs.2015.04.008

5. Ward M. Trans-scleral Gore-Tex fixation of Envista IOL. In: Chang D, editor. Advanced IOL Fixation Techniques: Strategies for Compromised or Missing Capsular Support. 1st ed. Thorofare, NJ: Slack Incorporated; 2019:235-243.

6. Veritti D, Grego L, Samassa F, Sarao V, Lanzetta P. Scleral fixation of a single-piece foldable acrylic IOL through a $1.80 \mathrm{~mm}$ corneal incision. $J$ Cataract Refract Surg. 2020;46(5):662-666. doi:10.1097/j.jcrs.0000000000000138

7. Su D, Stephens JD, Obeid A, et al. Refractive outcomes after pars plana vitrectomy and scleral fixated intraocular lens with Gore-Tex suture. Ophthalmol Retina. 2019;3(7):548-552. doi:10.1016/j.oret.2019.02.012

8. Kalevar A, Dollin M, Gupta RR. Opacification of scleral-sutured Akreos AO60 intraocular lens after vitrectomy with gas tamponade: case series. Retin Cases Brief Rep. 2020;14(2):174-177. doi:10.1097/ ICB.0000000000000634

9. Mojzis P, Studeny P, Werner L, Pinero DP. Opacification of a hydrophilic acrylic intraocular lens with a hydrophobic surface after air injection in Descemet-stripping automated endothelial keratoplasty in a patient with Fuchs dystrophy. J Cataract Refract Surg. 2016;42(3):485-488. doi:10.1016/j.jcrs.2016.02.004

10. Pan Q, Yang Z, Chen X, et al. Suturing technique for scleral fixation of toric intraocular lens in the traumatic aphakic eye with corneal astigmatism. Eur J Ophthalmol. 2019;29(1):100-105. doi:10.1177/ 1120672118769522

11. Burnham J, Tieu B Scleral-fixated Envista lens with Gore-Tex suture [American Academy of Ophthalmology web site]; November 16, 2017. Available from: https://www.aao.org/clinical-video/scleralfixated-envista-lens-with-gore-tex-suture. Accessed March 9, 2021.

12. Watane A, Botsford BW, Sood AB, et al. Scleral-sutured intraocular lens dislocations secondary to eyelet fractures. Am J Ophthalmol. 2021;221:273-278. doi:10.1016/j.ajo.2020.07.049

13. Pollmann AS, Lewis DR, Gupta RR. Structural integrity of intraocular lenses with eyelets in a model of transscleral fixation with the Gore-Tex suture. J Cataract Refract Surg. 2020;46(4):617-621. doi:10.1097/j. jcrs.0000000000000129 


\section{Publish your work in this journal}

Clinical Ophthalmology is an international, peer-reviewed journal covering all subspecialties within ophthalmology. Key topics include: Optometry; Visual science; Pharmacology and drug therapy in eye diseases; Basic Sciences; Primary and Secondary eye care; Patient Safety and Quality of Care Improvements. This journal is indexed on PubMed
Central and CAS, and is the official journal of The Society of Clinical Ophthalmology (SCO). The manuscript management system is completely online and includes a very quick and fair peer-review system, which is all easy to use. Visit http://www.dovepress.com/ testimonials.php to read real quotes from published authors.

Submit your manuscript here: https://www.dovepress.com/clinical-ophthalmology-journal 